\title{
Histoire des femmes et histoire religieuse de la France contemporaine : de l'ignorance mutuelle à l'ouverture
}

\section{Bruno Dumons}

\author{
(2) OpenEdition \\ Journals \\ Édition électronique \\ URL : https://journals.openedition.org/clio/66 \\ DOI : $10.4000 /$ clio.66 \\ ISSN : 1777-5299 \\ Éditeur \\ Belin \\ Édition imprimée \\ Date de publication : 1 avril 2002 \\ Pagination : 147-157 \\ ISBN : 2-85816-620-X \\ ISSN : $1252-7017$
}

Référence électronique

Bruno Dumons, « Histoire des femmes et histoire religieuse de la France contemporaine : de

l'ignorance mutuelle à l'ouverture », Clio. Histoire, femmes et sociétés [En ligne], 15 | 2002, mis en ligne le 08 février 2005, consulté le 28 avril 2022. URL : http://journals.openedition.org/clio/66 ; DOI : https://doi.org/10.4000/clio.66 


\section{Actualité de la recherche}

\section{Histoire des femmes et histoire religieuse de la France contemporaine : de l'ignorance mutuelle à l'ouverture*}

Bruno Dumons

"Écrire l'histoire des femmes" serait-elle une entreprise historiographique vouée à ne jamais prendre en compte la dimension religieuse de la personne comme de la société ? Le fait religieux peut-il être considéré comme un fait social ? Est-il vraiment devenu objet d'histoire ? Ces interrogations aux allures provocatrices concernent aussi l'histoire des femmes dans la mesure où elle s'est d'abord largement inscrite, pour le cas français, dans le champ de l'histoire sociale. Une histoire qui porte les stigmates d'une historiographie nationale et républicaine, aux relents parfois misogynes, renvoyant la religion à un passé révolu. Au lendemain de la Libération, l'historiographie contemporaine française, tributaire d'un héritage à la fois libéral et marxiste, est dominée par les vecteurs dynamiques de l'histoire que sont le peuple et le mouvement ouvrier, le plus souvent associés à l'irreligion et à la masculinité. Les femmes sont donc renvoyées aux "silences de l'histoire" tandis que le fait religieux tente difficilement de s'autonomiser et de s'institutionaliser au sein du récit historique.

* Le cadre de l'analyse s'attachera prioritairement au catholicisme sans que les conclusions apportées soient très différentes pour l'étude du protestantisme et du judaïsme.

CLIO, Histoire, Femmes et Sociétés, 2002, 15, pp. 147-157. 
Ainsi, il faut bien reconnaître que les deux champs que constituent l'histoire sociale et l'histoire religieuse n'ont pris que tardivement en compte la dimension sexuée de la société. Pourtant, ce peut être l'occasion d'élaborer des recherches aux interfaces du religieux et du social, souvent stimulantes pour chacun des deux domaines malgré leur éloignement supposé. Le regard croisé entre l'histoire des femmes et l'histoire religieuse, depuis environ une trentaine d'années, permettra de mieux saisir les moments de rupture qui ont caractérisé les travaux des contemporanéistes français mais également les efforts plus récents de collaboration et d'ouverture qui contribuent plus largement à une «opération de rénovation historiographique" mutuelle. Revisiter cette historiographie conduit de manière plus générale à une réflexion sur les objectifs d'une "histoire sociale du religieux" qui s'élabore à partir de recherches menées aux interfaces historiques puis conduit à plus long terme à un renouvellement des champs disciplinaires.

\section{Une histoire des femmes sans la religion est-elle possible ?}

Il faut pourtant bien en convenir, l'histoire des femmes en France ne s'est guère intéressée à la dimension du religieux. Dans une réflexion récente, Florence Rochefort reconnaissait d'ailleurs volontiers qu' "on a plus souvent réfléchi à la difficile relation du socialisme et du féminisme qu'à celle du féminisme et du christianisme qui est moins évidente à cerner» ${ }^{1}$. Cet état de fait est consécutif aux conditions de naissance de l'histoire des femmes comme discipline dont la gestation s'est faite en France au sein d'une histoire sociale qui se voulait totale et globale. Toute autre est l'approche historiographique en terme de genre où se ressent l'influence de la tradition anglo-saxonne qui a beaucoup moins tendance à écarter le fait religieux dans les rapports masculin-féminin. Ce courant n'a pénétré les recherches françaises que récemment.

Si nombre de clichés ont dévoilé une institution ecclésiale ayant pu compter sur les élites et les puissants de ce monde, ils ont également fait de la femme son fidèle soutien et son principal rempart. Michelet n'affir-

1 Rochefort $1998: 211$. 
me-t'il pas en 1860 que «la femme est une religion»? Cet esprit "naturellement» religieux des femmes conduit alors sur des chemins silencieux que les historiens hésiteront beaucoup à emprunter. Quant à l'histoire des femmes, très liée à ses débuts au féminisme militant, elle saisit avec des pincettes le fait religieux qu'elle associe à la domination des consciences et à l'exploitation des plus faibles. Le colloque de Saint-Maximin qui marque en 1984 l'affirmation de l'histoire des femmes en France, passe sous silence le facteur religieux ${ }^{2}$.

Une première rencontre s'est esquissée entre les femmes et la religion à partir de l'approche historique de la médecine. La présence des femmes, en particulier des religieuses, dans le personnel soignant a ponctuellement ouvert une brèche en direction du fait religieux avec Jacques Léonard, Yvonne Knibiehler et Olivier Faure ${ }^{3}$. La décennie 1990 ouvre alors en France le verrou qu'il fallait faire sauter pour que l'élément religieux intègre quelque peu l'histoire des femmes. La collection du même nom que dirigent Georges Duby et Michelle Perrot, accorde dans le volume consacré au XIX siècle plusieurs chapitres aux différentes confessions ${ }^{4}$. Si cette invitation ne suscite guère d'enthousiasme, notamment au sein des histoires du féminisme, elle sera toutefois reçue par certaines historiennes des femmes. Ainsi, Mathilde Dubesset et Michelle Zancarini n'hésitent pas à consacrer des pages très suggestives à la formation et à l'instruction congréganistes des femmes stéphanoises au début du siècle ${ }^{5}$. Parallèlement, l'historiographie américaine a, de manière plus précoce, pris en compte la dimension religieuse. Ainsi, Bonnie Smith dépeint pour la seconde moitié du XIX ${ }^{e}$ siècle des bourgeoises du Nord, pieuses et charitables, exerçant un rôle social déterminant $t^{6}$.

Il faut attendre la publication du deuxième numéro de la revue Clio en 1995 pour que la question religieuse soit abordée dans le champ de l'histoire des femmes, en France. D'autres passerelles ont également été posées

2 Perrot 1984.

3 Léonard 1977 ; Knibiehler 1984 ; Faure 1999.

4 Duby et Perrot 1991.

5 Dubesset et Zancarini-Fournel 1993.

6 Smith 1989. 
en direction du fait religieux par de jeunes historiennes. La solide étude d'Anne Cova sur la maternité en France au début du XX' siècle, montre comment les catholiques français ont exercé un rôle dans l'instauration d'une législation nationale, relayée par des institutions féminines confessionnelles. Elle vient d'ailleurs de livrer un ouvrage particulièrement stimulant sur l'action des femmes catholiques dans la protection de la maternité sous la Troisième République ${ }^{7}$. Nombre de ces associations féminines catholiques ont d'ailleurs été l'objet d'études spécifiques par des historiennes américaines, permettant ainsi de croiser la réflexion sur les rapports et les connexions entre histoire des femmes, des religions et de l'État-Providence.

Des thématiques comme celle de l'éducation sentimentale des jeunes filles ont suscité des recherches au sein desquelles les dimensions religieuses ont davantage été prises en compte ${ }^{8}$. Claude Langlois a souligné l'importance de la "féminisation du catholicisme» au XIX siècle. La période contestée de Vichy a donné lieu à une approche de la «culture catholique féminine» prônée par la Révolution Nationale?. La biographie de Germaine Poinso-Chapuis, femme d'État, prend en compte ses convictions chrétiennes ${ }^{10}$. Enfin, les travaux de Sylvie Chaperon sur le féminisme contemporain du "creux de la vague» mettent l'accent sur le rôle important joué par les femmes chrétiennes et leurs organisations sur la scène politique de l'après-guerre à $1968^{11}$. Reste que ce regard jeté sur les femmes et le religieux demeure encore un "point aveugle de l'histoire au féminin» malgré les invitations suggestives émises dans l'ouvrage dirigé par Karen Offen et l'appel judicieux, lancé par Françoise Thébaud, en vue d'une juste "articulation entre histoire religieuse et histoire des femmes» ${ }^{12}$. Toutefois, la synthèse livrée par Christine Bard a relevé avec bonheur le défi de faire une place au religieux dans son approche des femmes dans la

7 Cova 2000.

8 Houbre 1997.

9 Muel-Dreyfus 1996.

10 Knibielher 1998.

11 Chaperon 2000.

12 Offen 1991 ; Thébaud $1998:$ 150-151. 
société française du XXe siècle ${ }^{13}$, période pour laquelle Mathilde Dubesset vient de proposer une brève synthèse sur catholiques et protestantes ${ }^{14}$. Malgré ces efforts indéniables, les nouvelles orientations historiographiques de l'histoire des femmes n'ont pas encore totalement intégré le fait religieux et semblent encore souvent ignorer cette donnée pourtant nécessaire à tout regard historique sur les femmes dans la France contemporaine.

\section{L'histoire religieuse sans les femmes est-elle possible?}

Sur l'autre versant de l'histoire religieuse, un constat sévère pourrait être dressé, soulignant le peu d'intérêt que les femmes ont suscité au regard du bilan historiographique d'un champ, tiraillé jusqu'à l'ère conciliaire, entre une histoire de l'Église, à vocation apologétique et théologique, et une histoire politique et institutionnelle qui ont tendance à les exclure de leurs analyses. Le bilan est d'ailleurs maigre comme l'a établi la recension élaborée par l'équipe de Jean-Marie Mayeur en $1975^{15}$. Toutefois, l'histoire religieuse a beaucoup appris au contact d'autres sciences sociales du religieux comme la sociologie et l'anthropologie qui se sont engagée plus tôt dans des recherches sur les femmes.

Toujours secondaire est l'attention que les historiens du religieux ont posée sur les femmes comme objet historiographique. C'est le constat qu'établissait en 1995 Etienne Fouilloux dans un article de synthèse pour la revue Clio. Pourtant, une approche par les femmes était déjà en germe dans les enquêtes historiques de sociologie religieuse qui distinguaient les pascalisants et les messalisants selon les sexes. Obnubilés par la problématique de la "déchristianisation", en particulier ouvrière, les chercheurs se sont alors tardivement penchés sur cette distinction qui a permis d'observer une pratique féminine plus importante et de mettre l'accent sur la "féminisation" du catholicisme ${ }^{16}$. La mise en lumière de ce processus a

13 Bard 2001.

14 Dubesset 2000.

15 Mayeur 1975.

16 Langlois 1995. 
donné lieu à des travaux particulièrement riches sur l'éducation et l'enseignement des jeunes filles ainsi que des études sur le rôle exercé par les tiers-ordres et les congrégations religieuses féminines dans la société française ${ }^{17}$.

Durant la dernière décennie, l'intérêt a rejailli plus fortement avec des recherches abouties sur les associations et les mouvements féminins qui se déploient au début du XX' siècle autour du catholicisme social et de la jeunesse chrétienne mais également à propos de questions relatives à la transmission de la foi et à la théologie morale, avec la question de la procréation $^{18}$. D'autre part, le monde associatif et militant du début du siècle constitue un terreau essentiel pour la rencontre entre les femmes et les confessions religieuses ${ }^{19}$. Plus récemment encore, l'approche par la voie de la spiritualité a souligné le rôle majeur que des femmes ont pu exercer dans la transformation du sentiment religieux et l'invention de nouveaux modèles de vie consacrée ${ }^{20}$. La biographie est également un moyen de mettre en lumière des personnalités féminines marquantes dans le domaine de la foi. Cependant, le véritable élan semble revenir encore une fois à l'historiographie étrangère. L'histoire religieuse helvétique comme celle de I'Italie ont chacune intégré des approches problématiques sur les femmes et le religieux, à l'image notamment des travaux d'Urs Altermatt et de Roberta Fossati ${ }^{21}$. Mais l'apport le plus conséquent revient encore à l'historiographie anglo-saxonne qui, dès les années 1980, a investi le territoire hexagonal, avec notamment les travaux de Steven Hause et James McMillan, privilégiant une approche politique des ligues féminines, et surtout de l'australien Ralph Gibson dont le bilan sur «le catholicisme et les femmes en France au XIX siècle» a déjà été largement évoqué par Etienne Fouilloux ${ }^{22}$.

17 Langlois 1972 ; Mayeur 1977.

18 Delumeau 1992 ; Sevegrand 1995.

19 Diebolt 1993.

20 Benoist 2000 ; Perret 2000 ; Langlois 2001.

21 Altermatt 1994 ; Fossati 1997.

22 Fouilloux 1995. 
Comme le suggèrent les orientations de recherche proposées par Françoise Lautman, la collaboration avec la sociologie est à nouveau à l'ordre du jour, en particulier sur cette question des rapports entre "femmes et religion", conduisant à l'élaboration de problématiques communes en vue de renouveler les approches disciplinaires sur cette thématique. La psychanalyse a également constitué une voie dans laquelle ont eu lieu des confrontations de méthode pour interpréter des itinéraires spirituels et mystiques comme celui de Thérèse de Lisieux ${ }^{23}$. De manière plus générale, des synthèses récentes en histoire religieuse tentent désormais d'intégrer une approche spécifique à partir des femmes.

Revisitant récemment la question, Claude Langlois reconnaît que le bilan est encore bien maigre en ce qui concerne une histoire religieuse du fait féminin ${ }^{24}$. Il s'agit pour lui d'un "champ délaissé" qu'il convient de labourer rapidement, y compris avec les outils du "gender", tant les fruits qui en résulteront permettront de combler «une de ces lacunes dommageables» qui est le produit de «l'ignorance réciproque» entre l'histoire des femmes et l'histoire du fait religieux ${ }^{25}$. Il semble que l'appel ait été entendu de part et d'autre, conduisant à des initiatives concrètes difficilement imaginables, il y a encore quelques années ${ }^{26}$. La rencontre entre l'histoire des femmes et l'histoire religieuse peut offrir des perspectives nouvelles de recherches dans chacun des cas, contribuant à éclairer des angles morts historiographiques, fortement dommageables pour l'ensemble de la discipline historique. Ce travail semble désormais possible grâce à l'émergence de conditions favorables conduisant une génération d'historiens et d'historiennes à sortir de l'ignorance historiographique mutuelle et à envisager des convergences. Cette ouverture vers une «histoire sociale du reli-

23 Maître 1995.

24 Langlois 1997.

25 Langlois 2000a.

26 Lagrée et Monfrin 2000 ; Rochefort 2001. Dirigé par Gérard Cholvy, le dixième carrefour d'histoire religieuse a eu lieu à Bordeaux en juillet 2001 sur "la religion et les femmes». Sous la direction d'Anne Cova et de Bruno Dumons, un volume du Dictionnaire du monde religieux de la France contemporaine consacré aux femmes est en préparation chez Beauchesne. 
gieux" invite à dépasser ses frontières disciplinaires et historiographiques pour travailler aux interfaces des domaines du social et du religieux, de la sociologie et de l'histoire, du féminin et du masculin, permettant ainsi d'ouvrir les fenêtres de la discipline et de jeter des ponts vers d'autres champs des sciences humaines. Le temps serait-il désormais venu d'envisager une histoire des femmes ouverte au fait religieux comme celle d'une histoire religieuse au féminin ?

\section{Bibliographie}

ACCAMPO Elinor A., FUCHS Rachel G. et STEWART Mary Lynn (ed.), 1995, Gender and the Politics and Social Reform in France (18701914), Baltimore, The Johns Hopkins University Press.

ALTERMATT Urs, 1994, Le catholicisme au défi de la modernité. L'histoire sociale des catholiques suisses auX XIX" et XX" siècles, Lausanne, Payot.

ARNOLD Odile, 1984, Le corps et l'âme. La vie des religieuses au XIX siècle, Paris, Seuil.

BARD Christine, 1995, Les filles de Marianne. Histoire des féminismes (1914-1940), Paris, Fayard.

—, 2001, Les femmes dans la société française au XX siècle, Paris, Colin.

BENOIST Jacques, 2000, Le Sacré-Coeur et les femmes de 1870 à 1960, Paris, L'Atelier.

CADIER-REY Gabrielle (dir.), 2000, «Femmes protestantes au XIX`et au $\mathrm{XX}^{e}$ siècles", Bulletin de la Société de l'Histoire du Protestantisme Français, tome 146, janvier-mars.

CHAPERON Sylvie, 2000, Les années Beauvoir (1945-1970), Paris, Fayard.

CHEROUTRE Marie-Thérèse et CHOLVY Gérard, 1990, Scoutisme féminin et promotion féminine (1920-1990), Paris, Guides de France.

CHOLVY Gérard, 1997, Etre chrétien en France au XIX siècle (17901914), Paris, Seuil.

COVA Anne, 2000, "Au service de l'Église, de la patrie et de la famille». Femmes catholiques et maternité sous la Troisième République, Paris, L'Harmattan. 
DELUMEAU Jean (dir.) 1992, La religion de ma mère. Le rôle des femmes dans la transmission de la foi, Paris, Cerf.

DIEBOLT Evelyne, 1993, Les associations face aux institutions. Les femmes dans l'action sanitaire, sociale et culturelle (1900-1965), thèse de doctorat d'État, Université Paris VII.

DUBESSET Mathilde et ZANCARINI-FOURNEL Michelle, 1993, Parcours de femmes. Réalités et représentations. Saint-Étienne (18801950), Lyon, PUL.

-, 2000, "Des femmes en mouvement. Catholiques et protestantes au XX' siècle", Cahiers de Meylan, n 2, pp. 1-78.

DUBY Georges et PERROT Michelle (dir.), 1991b, Histoire des femmes, tome 4, Paris, Plon.

FAURE Olivier, 1999, "Les religieuses hospitalières entre médecine et religion en France au XIX siècle», Isabelle Von Bueltzingsloewen et Denis Pelletier (dir.), La charité en pratique. Chrétiens français et allemands sur le terrain social (XIX'-XX' siecles), Strasbourg, PUS, pp. 53-64. FAYET-SCRIBE Sylvie, 1990, Associations féminines et catholicisme. De la charité à l'action sociale (XIX'-XX' siècle), Paris, Éditions Ouvrières.

FOSSATI Roberta, 1997, Elites femminili e nuovo modelli religiosi nell'Italia tra Otto e Novecento, Urbino, Quattro Venti.

FOUILLOUX Etienne, 1995, "Femmes et catholicisme dans la France contemporaine. Aperçu Historiographique», Clio, n 2, pp. 319-329.

GIBSON Ralph, 1993, "Le catholicisme et des femmes en France au XIX siècle", Revue d'Histoire de l'Église de France, LXXIX, janvier-juin, pp. 63-93.

HAUSE Steven C. et KENNEY Anne R., 1981, "The Development of the Catholic Women's Suffrage Movement in France (1896-1822)", Catholic Historical Review, january, ${ }^{\circ} 1$, pp. 11-30.

HOUBRE Gabrielle, 1997, La discipline de l'amour. L'éducation sentimentale des filles et des garçons à l'âge du romantisme, Paris, Plon.

- (dir.), 1984, Cornettes et blouses blanches. Les infirmières dans la société française (1880-1980), Paris, Hachette.

KNIBIELHER Yvonne (dir.), 1998, Germaine Poinso-Chapuis. Femme d'Etat (1901-1981), Aix-en-Provence, Edisud. 
LAGREE Michel et MONFRIN Françoise, 2000, "Histoire religieuse et sciences humaines", Revue d'Histoire de l'Église de France, tome 86, $\mathrm{n}^{\circ}$ 217, juillet-décembre, pp. 519-538.

LANGLOIS Claude, 1972, "Les tiers-ordres dans le diocèse de Vannes", Claude Langlois et Paul Wagret, Structures religieuses et célibat féminin au XIX siècle, Lyon, CHC, pp. 11-115.

-, 1984, Le catholicisme au féminin. Les congrégations françaises à supérieure générale au XIX siècle, Paris, Cerf.

—, 1994-1995, "Catholicisme au féminin ou féminisation du catholicisme ? Délimiter ou définir : un nouveau champ de recherche en histoire et en sociologie", Annuaire de l'École Pratique des Hautes Études, $V^{e}$ section, tome 102, pp. 379-384.

—, 1995, “Toujours plus pratiquantes”. La permanence du dimorphisme sexuel dans le catholicisme français contemporain», Clio, $\mathrm{n}^{\circ} 2$, $\mathrm{pp}$. 229-260.

—, 1997, "Le catholicisme au féminin revisité», Alain Corbin, Jacqueline Lalouette et Michèle Riot-Sarcey (dir.), Femmes dans la Cité (18151871), Grâne, Créaphis, pp. 139-149.

—, 2000a, "Les champs délaissés», Revue d'Histoire de l'Église de France, tome $86, \mathrm{n}^{\circ} 217$, juillet-décembre, pp. 757-769.

—, 2000b, Les dernières paroles de Thérèse de Lisieux, Paris, Salvator.

—, 2001, "Spiritualité au féminin ? L'exemple du XIX siècle français", Christus, n 190 , avril, pp. 198-206.

LAUTMAN Françoise, 1997, "Introduction», Françoise Lautman (éd.), Ni Eve, ni Marie. Luttes et incertitudes des héritières de la Bible, Genève, Labor et Fides, pp. 7-17.

LEONARD Jacques, 1977, «Femmes, religion et médecine. Les religieuses qui soignent en France au XIX" siècle», Annales ESC, septembreoctobre, pp. 887-907.

MAITRE Jacques, 1995, "L'orpheline de la Bérésina». Thérèse de Lisieux (1873-1897), Paris, Cerf.

MAYEUR Françoise, 1977, L'enseignement secondaire des jeunes filles sous la Troisième République, Paris, FNSP.

- et GADILLE Jacques (dir.), 1980, Education et images de la femme chrétienne au début du XXX siècle, Lyon, L'Hermès. 
MAYEUR Jean-Marie (dir.), 1975, L'histoire religieuse de la France (XIX$X X$ siècles). Problèmes et méthodes, Paris, Beauchesne.

McMILlAN James F., 2000, France and Women (1789-1914). Gender, society and politics, Londres, Routledge.

MUEL-DREYFUS Francine, 1996, Vichy et l'eternel féminin, Paris, Seuil.

OFFEN Karen (ed.), 1991, Writing women's history : international perspectives, Bloomington, Indiana University Press.

PERRET Marie-Antoinette, 2000, Une vocation paradoxale. Les instituts séculiers féminins en France (XIX-XX' siècles), Paris, Cerf.

PERROT Michelle (dir.), 1984, L'histoire des femmes est-elle possible?, Marseille, Rivages.

POUJOL Geneviève et ROMER Madeleine (dir.), 1996, Dictionnaire biographique des militants (XIX'-XX siècles), Paris, L'Harmattan.

ROCHEFORT Florence, 1998, "La prostituée et l'ouvrière. Approches protestantes et catholiques du féminisme sous la Troisième République», Françoise Lautman (éd.), Ni Ève, ni Marie. Luttes et incertitudes des héritières de la Bible, Genève, Labor et Fides, pp. 211-229.

—, 2001, "Émile Poulat et "l'histoire des femmes"», Valentine Zuber (dir.), Emile Poulat. Un objet de science, le catholicisme, Paris, Bayard, pp. 80-84.

ROUX Jacqueline, 1995, Sous l'étendard de Jeanne. Les fédérations diocésaines de jeunes filles (1904-1945), Paris, Cerf.

SARTI Odile, 1992, The Ligue Patriotique des Françaises (1902-1933). A Feminine Response to the Secularization of French Society, New York, Garland Publishing.

SEVEGRAND Martine, 1995, Les enfants du bon Dieu. Les catholiques français et la procréation au XX siècle, Paris, Albin Michel.

SMITH Bonnie G., 1989, Les bourgeoises du Nord (1850-1914), Paris, Perrin.

SOHN Anne-Marie et THELAMON Françoise (dir.), 1998, L'histoire sans les femmes est-elle possible ?, Paris, Perrin.

THÉBAUD Françoise, 1998, Ecrire l'histoire des femmes, Fontenay, ENS Éditions.

TURIN Yvonne, 1989, Femmes et religieuses au XIX siècle. Le féminisme «en religion», Paris, Nouvelle Cité. 